\title{
Anxiety as a Developmental Disorder
}

\author{
E David Leonardo ${ }^{1,3}$ and Rene Hen ${ }^{\star, 1,2,3}$ \\ ${ }^{1}$ Department of Psychiatry, Columbia University, New York, NY, USA; ${ }^{2}$ Department of Neuroscience, Columbia University, \\ New York, NY, USA; ${ }^{3}$ Division of Integrative Neuroscience, The New York State Psychiatric Institute, New York, \\ NY, USA
}

There is increasing recognition that many psychiatric disorders including anxiety disorders are neurodevelopmental in their origins. Here, we review and integrate data from human studies and from animal models that point to a critical period during which neural circuits that mediate anxiety develop. We then postulate that this highly plastic critical period is a time of heightened responsiveness that is particularly susceptible to adverse events. We discuss these concepts in the context the current heightened interest in gene by environment interactions in psychiatric illness emphasizing the importance of the temporal relationship between gene action and environmental milieu.

Neuropsychopharmacology Reviews (2008) 33, 134-140; doi:10.1038/sj.npp.1301569; published online 12 September 2007

Keywords: development; animal models; critical period; gene-environment; anxiety; serotonin

\section{INTRODUCTION}

Anxiety is an emotion that is easily understood but eludes precise definition. In the context of this review, we will define anxiety as a state of cognitive and behavioral preparedness that an organism mobilizes in response to a future or distant potential threat. In its non-pathological form of anxiety can be divided into two categories: (1) state anxiety, which is an acute adaptive response of heightened vigilance and arousal that enables an organism to navigate an unfamiliar environment of unknown danger and (2) trait anxiety which is a measure of an individual's baseline reactivity or tendency to generate anxious response. In its pathological form, anxiety is a maladaptive state that impairs the ability of an organism to respond optimally to its environment. Disorders of anxiety are divided into six discrete categories by the Diagnostic and Statistical Manual of the American Psychiatric Association (American Psychiatric Association. and American Psychiatric Association Task Force on DSM-IV, 2000). These include generalized anxiety disorder (GAD), social phobia, simple phobia, panic disorder, post-traumatic stress disorder, and obsessivecompulsive disorder. Anxiety disorders are highly prevalent and are associated with high levels of morbidity and mortality as well as high cost. It is estimated that anxiety disorders may affect up to $20 \%$ of the population at some

${ }^{*}$ Correspondence: Dr R Hen, Department of Psychiatry and Neuroscience, Columbia University, The New York State Psychiatric Institute, 1051 Riverside Drive, Unit 87, New York, NY, USA, Tel: +1212543 5328, Fax: +1212543 5410, E-mail: rh95@columbia.edu

Received 27 June 2007; revised 9 August 2007; accepted 13 August 2007 point in their lifetime with an annual estimated cost of $\$ 44$ billion dollars in the United States alone (Greenberg et al, 1999).

A developmental approach is particularly relevant to anxiety disorders as they are among the first psychiatric disorders to manifest. For instance, while the mean age of onset for depression is 29 , the mean age of onset for an anxiety disorder is 11 (Kessler et al, 2005). This early onset is consistent with the finding that individual levels of trait anxiety are established at an early age and are fairly constant over a lifetime. Thus, both trait anxiety and anxiety disorders are likely to be determined by early developmental processes or events that affect the way an individual brain is 'wired' (Kagan and Snidman, 1999; Schwartz et al, 1999; Van Ameringen et al, 1998).

We know that there is a significant genetic component to the way anxiety circuits develop, with twin studies in humans suggesting that $30-40 \%$ of the variance in GAD can be accounted for by genetic factors (Hettema et al, 2001; Sullivan et al, 2000). However, the genetic component is not specific for anxiety as the genetic risk for developing generalized anxiety is largely overlapping with the genetic risk for major depression (Kendler et al, 1992; Ninan and Berger, 2001; Roy et al, 1995). Thus, the phenotypic differences observed between the two disorders is likely driven by interactions between a common set of genetic risk factors and differing early environmental conditions (Kendler et al, 1992; Roy et al, 1995).

Prior to the publication of landmark studies like those by Caspi et al (2002) demonstrating how early maltreatment interacts with the monoamine oxidase A genotype to yield antisocial behavior and how the 5-HTT transporter interacts with adverse early adult events to increase risk of 
depression (Caspi et al, 2003), our understanding of how early environmental conditions might differentially impact the expression of underlying genetic risk had been limited, as these two fields of inquiry had been the purvey of two separate disciplines. Indeed, genetic studies generally look for effects above and beyond that of the environment, while epidemiological studies of risk typically look for environmental effects that emerge despite any underlying genetic variability in the study population. Thus, neither approach alone is capable of providing a full explanation of the environmental and genetic contributions to behavior. This is because genes do not generate behaviors directly. Rather, they act in developmental pathways that first generate neurons and then circuits and finally systems that mediate behavioral responses. The genetic program therefore unfolds in a predictable manner that samples the surrounding environment and is in turn shaped by it. In such a model, one would predict that the effect of any given environment will depend on the developmental program that is unfolding at the time. Thus, gene by environment interactions is perhaps more appropriately conceived of as gene by environment by time interactions, with some time periods being more susceptible to environmental manipulation than others.

The idea that environmental exposures occurring during periods of heightened brain plasticity may be of particular importance in establishing baseline brain function is encompassed in the concept of developmental critical periods (Hensch, 2004; Knudsen, 2004). Here, we apply the concept of developmental critical periods to understanding the origins of pathological anxiety and then illustrate these concepts with specific examples of specific gene $\times$ environment interactions that shape the circuitry underlying anxiety.

\section{Development and Critical Periods}

Patterns of adult behavior are shaped by the complex aggregate of responses made by an organism during development. Responses are encoded by neural systems that are built from simpler neural circuits, all of which have distinct developmental trajectories.

As the brain develops and neural systems mature, they become receptive to and interact with particular aspects of the environment, helping the organism build its behavioral repertoire. Such developmental sequences and behavioral patterns can be observed shortly after birth. For example, many human infants reproducibly demonstrate distress in response to strangers at between 7 and 9 months of age and show heightened sensitivity to strangers during this period (Bowlby, 1969; Emde et al, 1976). The onset of the stranger anxiety is fairly rapid and usually lasts only a few months. By the age of 2 years, children establish cohesive patterns of response to novel environments as evidenced by measures of behavioral inhibition which are stable over many years (Hirshfeld et al, 1992; Kagan et al, 1988, 1995, 1998), and predicts future risk of anxiety disorders (Kagan and Snidman, 1999). The precise and reproducible timing of such events during development generates the opportunity for unique gene by time by environment interactions that ultimately determine the mature functioning of the circuitry and the behaviors it mediates. Such defined time windows have been called critical or sensitive periods (Hensch, 2004; Knudsen, 2004).

The existence of highly plastic brief, defined periods of development whose outcome has long-term functional consequences, make these time-points particularly vulnerable for disruption by environmental influences. Similarly, this concept suggests that exposure to the same environmental influence at different points in time may result in dramatically different outcomes.

\section{Evidence from Human Anxiety}

Although the DSM classifies anxiety into six major classes, by design, these classes are devoid of any etiological significance. As such it is difficult to make broad statements across anxiety disorders. Attempts to classify anxiety in ways that have etiological relevance have come from multiple sources including factor analysis, genetic studies, risk factor studies, and treatment research. These approaches give somewhat different results, although a few broad themes emerge. For example, specific phobias appear to be distinguishable from other forms of anxiety on several fronts, including shared vulnerability models, and response to selective serotonin re-uptake inhibitors (SSRI's). Specific phobias have also been distinguished from GADs using factor analysis (Hettema et al, 2005; Kendler et al, 2003), which also places generalized anxiety and depression on the same factor, separate from other forms of anxiety. For simplicity, our discussion here will focus primarily on factors relevant to generalized anxiety.

Early life anxiety, particularly generalized anxiety, appears to predict both anxiety and depression later in life (Breslau et al, 1995; Parker et al, 1999; Weissman et al, 2005). Such a prediction is not surprising, given the evidence that both disorders share underlying genetic vulnerabilities. How environmental and temporal factors affect the expression of this underlying vulnerability is less clear. One leading theory is the stress-diathesis model, in which negative 'stressful' events interact with underlying vulnerabilities to produce illness.

Insights into the mechanism and possible temporal importance of these negative events comes from structural equation modeling studies of the stress-diathesis model which suggests at least two ways in which negative events may operate. One alternative is that negative events are mediators that activate or amplify underlying vulnerabilities. In this mediator model, stressful events act on the individual to change their vulnerability to, or risk for pathology. Another alternative is that the environment is a moderator that acts directly on pre-existing vulnerabilities to generate pathological responses (Figure 1). These models are not mutually exclusive. Indeed, it appears that risk factors early in life mediate the risk for anxiety and later depression, while later psychological factors may moderate the development of depression (Chorpita et al, 1998; Cole and Turner, 1993; Hammen et al, 1988; Nolen-Hoeksema et al, 1992). Together, these models are consistent with the possibility that environmental factors interact with immature emotion circuits during a critical window in early development in a way that affects the likelihood of an individual to developing anxiety or depression (mediator) whereas stressors occurring later in 


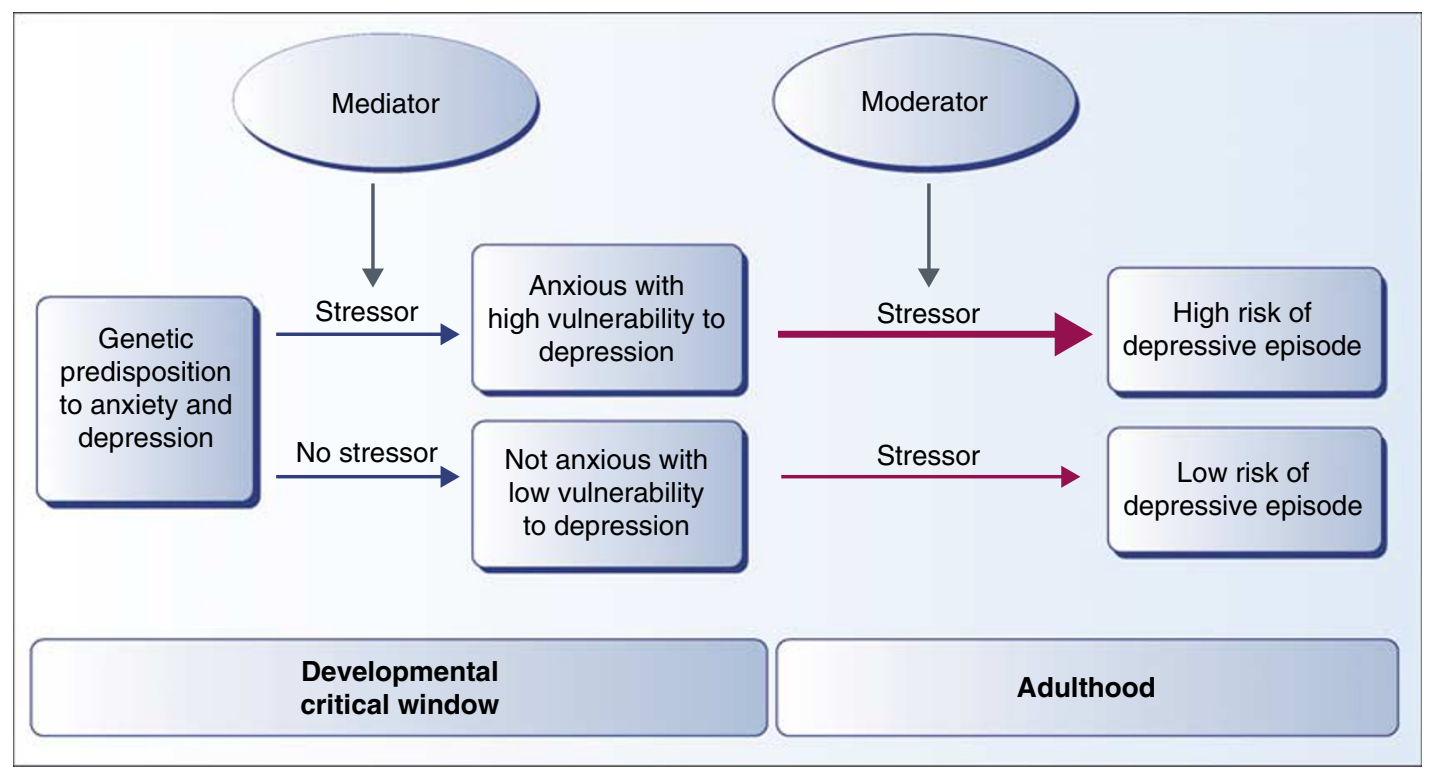

Figure I Mediators and moderators in psychiatric illness. Diagram depicts possible outcomes for an individual with genetic predispositions to anxiety or depression. (I) A stressor or adverse environment during an early critical period leads to anxiety and mediates (alters or sets) vulnerability to depressive episodes later in life. Thus, individuals experiencing the stressor may become anxious and are at increased risk for a depressive episode later in life while those not experiencing the stressor are not. (2) A stressor later in life, after the critical period acts on pre-existing vulnerability (moderator) to generate the expression of symptoms such that for any given stressor equivalent stressor, highly vulnerable individuals have higher rates of depressive episodes than less vulnerable individuals.

life act on a pre-determined likelihood to induce symptoms (moderator). Thus, the same genes interacting with similar environmental factors at different times can result in different outcomes.

There has been some recent debate in the literature about how the relationship between generalized anxiety and depression should be characterized in the next generation of the DSM (DSM-V) (Kessler and Wittchen, 2002; Wittchen et al, 2000). A recent study using prospectively collected data examined childhood risk for both generalized anxiety and depression in a single cohort (Moffitt et al, 2007). In this study, the authors examined childhood risk factors for people who developed either GAD only, major depressive disorder (MDD) only, or co-morbid GAD + MDD. Interestingly, the authors find that pure MDD was not strongly associated with environmental risk factors during childhood while pure GAD was associated with several risk factors across multiple domains of risk in early childhood. Patients with co-morbid GAD-MDD had a risk factor profile that was similar to the GAD alone group. Results from this study are consistent with the ideas that the presence of risk factors in early life mediate an increase risk of both generalized anxiety and depression, while environmental factors later in life moderate the emergence of depression. These results are not merely of academic interest as patients with GAD or co-morbid GAD-MDD were significantly more impaired than their MDD alone counterparts. Given the shared genetic burden of the two disorders, one might speculate that interventions early in life aimed at reducing environmental risk factors might shift the balance of the phenotypic expression of the genetic risk from GAD or co-morbid GAD-MDD to that of MDD alone.

Thus, data from human studies suggest that a person's baseline anxiety levels are likely due to an interaction between genetic and environmental factors occurring early in life. Through what mechanism do these factors act? How does the organism translate its response during this highly plastic period into a long-term relatively stable trait? Possible answers to these questions come from studies of anxiety in animal models.

\section{Evidence from Animal Studies}

Modeling anxiety and depression-like behavior in animals relies on the remarkable similarity that exists across species at the anatomical, molecular, physiological, and circuit level (Chrousos, 1998; Cooper et al, 2003). Neuromodulatory systems such as dopamine and serotonin are highly homologous in their function, helping to process and integrate information from the environment to generate context-dependent, species-specific behavior that is required by the organism. Similarities in the final circuitry at the adult level points to highly similar developmental processes across species. Thus, it is reasonable to assume that understanding the molecular mechanisms by which early environmental influences alter circuit development in rodents will tell us something about the development of anxiety in humans.

A number of brain structures have been implicated in anxiety circuits. These include the amygdala, temporal, and prefrontal cortices. In addition, there is evidence that monoaminergic modulation plays a significant role and that the stress-hormone response system is involved in the pathophysiology of anxiety and mood-related disorders. Perhaps some of the best evidence to date that provides insight into specific mechanisms through which the environment can act to alter anxiety-circuits comes from studies involving manipulations of the serotonergic system. A few of the more salient studies are discussed below. 
The serotonin-1A receptor and the serotonin transporter (5-HTT). The 5-HT1A receptor has been implicated in mediating the effects of serotonergic agents in anxiety and depression. Selective desensitization of 5-HT1A autoreceptors in the raphe has been postulated to be a key change that allows antidepressant action (Albert and Lemonde, 2004), although effects on postsynaptic receptors in the hippocampus likely play a role. Mice that have been genetically engineered to be lacking the 5-HT1A receptor show increased anxiety in a number of tests, including hippocampal dependent tasks (Klemenhagen et al, 2006; Parks et al, 1998; Ramboz et al, 1998).

One criticism of traditional knockout approaches has been that it is difficult to parse out developmental effects of a gene of interest from its adult functional role. In order to test for a possible developmental role of the 5-HT1A receptor in establishing anxiety, Gross et al (2002) generated a conditional knockout mouse that allowed for the selective rescue of postsynaptic $1 \mathrm{~A}$ receptors in a temporally regulatable manner (Santarelli et al, 2003). Using this strategy, they demonstrated that initiating expression of the receptor after p21 (reaching wt levels about 1 week later) resulted in anxiety levels that are indistinguishable from knockout animals. Conversely, earlier expression the receptor (with significant levels appearing by p15) resulted in animals with anxiety levels that were indistinguishable from wild-type animals. This finding indicates that 5-HT is essential to the establishment of normal anxiety-modulating circuits in the brain during postnatal development, likely in the third and fourth postnatal week.

Additional evidence for a developmental role of serotonin in establishing baseline levels of anxiety comes from studies of mice lacking the serotonin transporter, as well as from mice treated in early postnatal life with fluoxetine. One paradox in the field has been that 5-HTT knockout mice exhibit higher levels of anxiety than their wild-type counterparts. This result is counter-intuitive as selective serotonin reuptake inhibitors are highly effective at treating anxiety disorders in adults. The result also suggested the possibility that the effects of lacking 5-HTT function in development may be different from the effects of inhibiting 5-HTT function in the adult. In order to test this possibility, young mice (postnatal days 4-21) were treated with fluoxetine, producing in effect a pharmacological equivalent of a temporally restricted knockout (Ansorge et al, 2004). The results from this experiment demonstrated that blockade of the serotonin transporter with fluoxetine during days 4-21 was sufficient to produce a deficit in exploratory behavior in a novel environment that mimicked a deficit seen in mice with lifelong deletion of the transporter (see Figure 2). Finally, the possible role of early life stress on abnormal emotion-related behaviors in 5-HTT knockout mice during and early time window has been examined. $\mathrm{Wt}$ or 5-HTT knockout mice were subjected to footshocks during days $7-13$, then behaviorally tested the animals as adults. Interestingly, they did not find any effects of stress on anxiety-like measures in these animals (Carroll et al, 2007). Taken together, the 5-HT1A rescue experiments and 5-HTT mice treated with fluoxetine in the early postnatal period suggest that serotonin plays a critical role in the maturation of circuits relevant to anxiety prior to the third postnatal week. The negative result on the interaction between footshock on days p7-13 and later expression of anxiety suggest that the important window is after the end of the second week. We would therefore predict that a repeat of the footshock experiment at a later window (p1521) would show lasting effects on anxiety behavior which might be altered in these 5-HTT-deficient mice.

The developmental role for serotonin in circuit maturation is not restricted to circuits critical for anxiety. For example, serotonin is known to play a critical role in the development of whisker barrel fields in the somatosensory cortex of the mouse. The critical period for this process appears to be postnatal days 1-6 (Fox, 1992). At a later stage, Nakamura et al (2006) have identified TPH1, an enzyme involved in the rate-limiting step for serotonin synthesis as playing a role in the maturation of sensorimotor gating. Using pharmacological blockade, the authors demonstrate that TPH1 is specifically required during postnatal days 21-24 for proper maturation of the circuit. Thus, it is clear that serotonin has distinct, clearly defined roles in circuit maturation at different times during development.

Finally, it is important to emphasize that while most of the work done to date is in the serotonergic system, other neurotransmitter systems demonstrate postnatal developmental time courses that suggest possible roles in shaping late developing circuits relevant for behavior. For example, norepinephrine levels in the developing rat brain do not reach full adult levels in the cortex until the fifth or sixth postnatal week (Murrin et al, 2007). Similarly, dopaminergic fibers in the rat prefrontal cortex show sparse projections in the second postnatal week, with progressive in-growth that continues into the early adult period (Benes et al, 2000).

Postnatal brain development during the sensitive period for maturation of anxiety circuits. As discussed in the preceding section, there is accumulating evidence suggesting that signaling via serotonin during a period comprising the second and third weeks of life in mice appear be a critical period for the development of circuits that mediate anxiety. Such a description implies a time of heightened plasticity in response to relevant environmental stimuli. In order to understand the possible mechanisms at work, we need an understanding of the developmental events that are occurring during this period.

The second and third postnatal week in the rodent are a time of dramatic change during which the animal transitions from being fully dependent on its mother for nutrition to establishing the ability to find food independently. It is during this time behaviors relevant to anxiety emerge. Animals develop reactions to novelty and develop exploratory activity, behaviors critical to developing as independent adults. Early in this postulated critical window, by about day 15, rats transition from predominantly crawling, to the adult-like quadriped walking (Altman and Sudarshan, 1975; Westerga and Gramsbergen, 1990). This transition happens during a peak of locomotor activity as animals demonstrate a dramatic rise in locomotion between postnatal days 10 and 15. Interestingly, the transition to quadriped walking is accompanied by a decrease in locomotion and an increase in exploratory activity as 
Critical Period for the development of anxiety:

The role of the serotonergic system

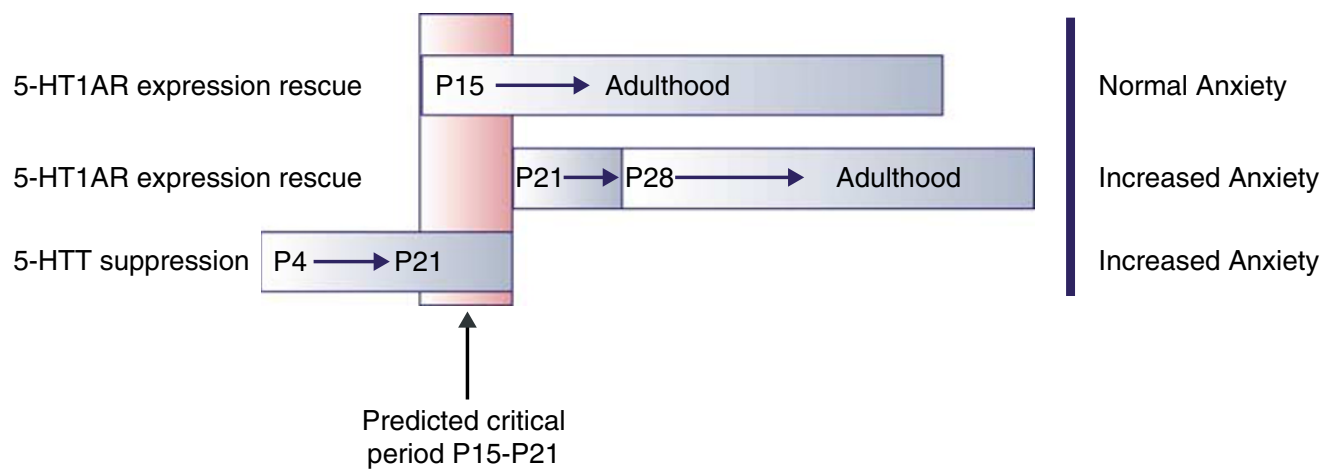

Figure 2 Figure summarizes data supporting a critical role for serotonergic signaling in establishing normal anxiety circuits. (top) Expression of 5-HTIAR in a knockout background beginning at day 15 is sufficient to rescue normal behavior, while graded re-expression of the receptor beginning a P2I results in an anxious phenotype (middle). Finally, a functioning serotonin transporter between P4 and $2 \mathrm{I}$ is necessary for the development of normal anxiety as suppression during this period elicits a sustained anxious phenotype (bottom). These results suggest that a critical period exists centered on the PI5-2I period.

measured in a hole-board apparatus. Indeed, an inverse relationship between exploration and locomotion is observed between days 20 and 45, with a peak of exploratory activity occurring between days 20 and 30 and then declining to day 45 (Ba and Seri, 1993). These time points also coincide with the peak levels of serotonin in the brain, which are low in the early postnatal period, increasing to a peak between days 21 and 30 before dropping down to adult levels (Loizou, 1972).

The rise in exploratory activity occurs in the context of two additional emerging behaviors of relevance to anxiety. First, habituation to novelty, defined as decreased locomotor activity in response to repeated exposure to an environment, does not exist at day 15 but emerges by day 20. Similarly, emotional reactivity as measured by defecation in a novel environment emerges at day 20. Thus, the behavioral evidence suggests that the circuits that mediate anxious responses to novelty are rapidly maturing between postnatal days 15 and 20. This is precisely the time window during which manipulations of the serotonergic system appear to result in permanent alterations of baseline levels of anxiety for adult animals as we described above for 5-HT1AR rescue and 5-HTT mice treated with postnatal fluoxetine. Consistent with these data are results from our laboratory on the timeline for emergence of the constitutive 5-HT1AR knockout mouse phenotype. Knockout and wild-type mice show no behavioral differences at PND 15 with a difference in anxiety measures appearing on day 21 (Klemenhagen et al, unpublished results). In addition to being the time frame for the emergence of anxiety-like behavior, this time-period coincides with a dramatic upregulation of expression of the 5 -HT1A receptor in the CA1 region of the hippocampus (Richardson-Jones et al unpublished results), a structure that has been implicated in mediating anxiety-like behavior (Gray and McNaughton, 2000).

How do these behavioral data mesh with our understanding of the structural and molecular changes that are occurring at this time? It is clear that in order to have emotional reactivity to novelty, the animals must be able to engage their environment and integrate sensory information into long-term representations. One interpretation of lack of habituation at day 15 is that animals are unable to create appropriate representations of novel spaces ( $\mathrm{Ba}$ and Seri, 1993). In this way, these animals are similar to adult animals with bilateral hippocampal lesions which also show deficits in habituation and anxiety-like behavior. Indeed, the hippocampus develops rapidly during the third and fourth postnatal week, with many hippocampal-mediated tasks reaching full adult proficiency during that 2-week window (Beauregard et al, 1995).

As mentioned above, the hippocampus is a relatively late maturing structure. Indeed, at postnatal day 5 in the in the rat, the CA1 pyramidal neurons, (the main output neurons of the hippocampus proper) have a single long apical dendrite and a short rarely branched basal dendrite (Pokorny and Yamamoto, 1981). Few spines are seen on these dendrites. The dendrites mature over the next several weeks, with different aspects of the dendrites maturing at different rates. For example, during the second and third week, at the end of which time the animals are weaned, apical dendrites within the stratum radiatum and the stratum lacunosum reach their mature form while more distal portions continue to mature (Pokorny and Yamamoto, 1981). During this same time period, gabaergic interneurons are setting up their mature patterns of connectivity. For example, in CA1 and CA3 gabaergic terminals increase 4-6-fold between PND7 and postnatal day 21. In addition, Kv3 potassium channels, which are critical to the fast spiking of parvalbumin positive interneurons, appear in the first postnatal week, with a rapid increase in the second postnatal week, reaching mature levels by postnatal day 21 (Danglot et al, 2006; Tansey et al, 2002).

In summary, the second and third postnatal week comprise a period of rapid development in neurocircuitry, during which the mature patterns of hippocampal connectivity are being established. This time period corresponds to the time during which rodents are beginning to sample their environment on their own for the first time, providing the opportunity for environmental factors to shape the behavioral response of the animal to future environmental stimuli in ways that are not possible at later, less plastic time points. 


\section{FUTURE DIRECTIONS}

The importance of gene $\times$ environment interaction in the etiology of psychiatric illness has never been clearer, and we are for the first time achieving success understanding the mechanisms through which some of these interactions take place. To build on this success, we need a full understanding of how circuits develop and how perturbations during critical periods (mediators) affect circuit formation. We then need to develop an understanding of how these aberrant circuits respond to the environment later in life (moderators). In work with animal models, this will mean combining genetic strategies that allow for temporal and spatial control of gene expression with strategies that challenge animals in different environments at different developmental time points. For example, in the case of postnatal fluoxetine-treated animals (pnd 5-20) mentioned above in which animals appear anxious as adults, it would be interesting to see how these animals respond to additional challenges as adult. Are they more susceptible to chronic stress paradigms or to learned helplessness paradigms than littermate controls? Does the early anxiety induced by SSRI treatment increase susceptibility to depression based paradigms, therefore relating the animal work back to the known links between anxiety and depression in humans? Finally, how do these animals respond to pharmacological challenges as adults? Will these animals respond to treatment with SSRI's later in life or will they be treatment resistant compared to controls? By addressing gene and environmental effects through the lifespan, we will be better able to model human disease, and therefore achieve a better understanding of the underlying pathophysiology.

\section{DISCLOSURE/CONFLICT OF INTEREST}

Dr René Hen receives compensation as a consultant for BrainCells Inc., PsychoGenics Inc., Memory Pharmaceuticals, Roche, Astra Zeneca, and Lundbeck in relation to the generation of novel antidepressants. Dr E David Leonardo has no financial interests to disclose.

\section{REFERENCES}

Albert PR, Lemonde S (2004). 5-HT1A receptors, gene repression, and depression: guilt by association. Neuroscientist 10: 575-593.

Altman J, Sudarshan K (1975). Postnatal development of locomotion in the laboratory rat. Anim Behav 23: 896-920.

American Psychiatric Association, American Psychiatric Association. Task Force on DSM-IV (2000). Diagnostic and Statistical Manual of Mental Disorders: DSM-IV-TR, 4th edn. American Psychiatric Association: Washington, DC, 37: 943 pp.

Ansorge MS, Zhou M, Lira A, Hen R, Gingrich JA (2004). Earlylife blockade of the 5-HT transporter alters emotional behavior in adult mice. Science 306: 879-881. Demonstrates how even a transient loss of gene function during a critical period in development can have long-term behavioral consequences. The data presented here nicely complement data on the effects of human 5-HTT polymorphisms on early life adverse events.

Ba A, Seri BV (1993). Functional development of central nervous system in the rat: ontogeny of nociceptive thresholds. Physiol Behav 54: 403-405.
Beauregard M, Malkova L, Bachevalier J (1995). Stereotypies and loss of social affiliation after early hippocampectomy in primates. Neuroreport 6: 2521-2526.

Benes FM, Taylor JB, Cunningham MC (2000). Convergence and plasticity of monoaminergic systems in the medial prefrontal cortex during the postnatal period: implications for the development of psychopathology. Cereb Cortex 10: 1014-1027.

Bowlby J (1969). Attachment and Loss: Vol. 1. Attachment. Basic Books: New York. pp 321-330.

Breslau N, Schultz L, Peterson E (1995). Sex differences in depression: a role for preexisting anxiety. Psychiatry Res 58: $1-12$.

Carroll JC, Boyce-Rustay JM, Millstein R, Yang R, Wiedholz LM, Murphy DL et al (2007). Effects of mild early life stress on abnormal emotion-related behaviors in 5-HTT knockout mice. Behav Genet 37: 214-222.

Caspi A, McClay J, Moffitt TE, Mill J, Martin J, Craig IW et al (2002). Role of genotype in the cycle of violence in maltreated children. Science 297: 851-854.

Caspi A, Sugden K, Moffitt TE, Taylor A, Craig IW, Harrington H et al (2003). Influence of life stress on depression: moderation by a polymorphism in the 5-HTT gene. Science 301: 386-389.

Chorpita BF, Brown TA, Barlow DH (1998). Perceived control as a mediator of family environment in etiological models of childhood anxiety. Behav Ther 29: 457-476.

Chrousos GP (1998). Stressors, stress, and neuroendocrine integration of the adaptive response. The 1997 Hans Selye Memorial Lecture. Ann NY Acad Sci 851: 311-335.

Cole DA, Turner Jr JE (1993). Models of cognitive mediation and moderation in child depression. J Abnorm Psychol 102: 271-281.

Cooper JR, Bloom FE, Roth RH (2003). The Biochemical Basis of Neuropharmacology, 8th edn. Oxford University Press: Oxford, New York, 7: $40 \mathrm{pp}$.

Danglot L, Triller A, Marty S (2006). The development of hippocampal interneurons in rodents. Hippocampus 16: 1032-1060.

Emde RN, Harmon RJ, Gaensbauer TJ (1976). Emotional Expression in Infancy: A Biobehavioral Study. International Universities Press: New York, 11: 19 pp.

Fox K (1992). A critical period for experience-dependent synaptic plasticity in rat barrel cortex. J Neurosci 12: 1826-1838.

Gray JA, McNaughton N (2000). The Neuropsychology of Anxiety: An Enquiry into The Functions of The Septo-Hippocampal System, 2nd edn. Oxford University Press: Oxford, New York, 16: $424 \mathrm{pp}$.

Greenberg PE, Sisitsky T, Kessler RC, Finkelstein SN, Berndt ER, Davidson JR et al (1999). The economic burden of anxiety disorders in the 1990s. J Clin Psychiatry 60: 427-435.

Gross C, Zhuang X, Stark K, Ramboz S, Oosting R, Kirby L et al (2002). Serotonin1A receptor acts during development to establish normal anxiety-like behaviour in the adult. Nature 416: 396-400. Demonstrates the requirement for 5-HT1AR function in the forebrain during early postnatal development in order to establish normal anxiety circuits. Also demonstrates that expression of the receptor in the adult animal is unable to reverse the developmental phenotype.

Hammen C, Adrian C, Hiroto D (1988). A longitudinal test of the attributional vulnerability model in children at risk for depression. Br J Clin Psychol 27: 37-46.

Hensch TK (2004). Critical period regulation. Annu Rev Neurosci 27: $549-579$.

Hettema JM, Neale MC, Kendler KS (2001). A review and metaanalysis of the genetic epidemiology of anxiety disorders. Am J Psychiatry 158: 1568-1578.

Hettema JM, Prescott CA, Myers JM, Neale MC, Kendler KS (2005). The structure of genetic and environmental risk factors for anxiety disorders in men and women. Arch Gen Psychiatry 62: 182-189. 
Hirshfeld DR, Rosenbaum JF, Biederman J, Bolduc EA, Faraone SV, Snidman N et al (1992). Stable behavioral inhibition and its association with anxiety disorder. J Am Acad Child Adolesc Psychiatry 31: 103-111.

Kagan J, Reznick JS, Snidman N (1988). Biological bases of childhood shyness. Science 240: 167-171. Longitudinal study that demonstrates the stability of behavioral inhibition as initially assessed at age 2 over time.

Kagan J, Snidman N (1999). Early childhood predictors of adult anxiety disorders. Biol Psychiatry 46: 1536-1541.

Kagan J, Snidman N, Arcus D (1995). The role of temperament in social development. Ann NY Acad Sci 771: 485-490.

Kagan J, Snidman N, Arcus D (1998). Childhood derivatives of high and low reactivity in infancy. Child Dev 69: 1483-1493.

Kendler KS, Neale MC, Kessler RC, Heath AC, Eaves LJ (1992). Major depression and generalized anxiety disorder. Same genes, (partly) different environments? Arch Gen Psychiatry 49: 716-722.

Kendler KS, Prescott CA, Myers J, Neale MC (2003). The structure of genetic and environmental risk factors for common psychiatric and substance use disorders in men and women. Arch Gen Psychiatry 60: 929-937.

Kessler RC, Berglund P, Demler O, Jin R, Merikangas KR, Walters EE (2005). Lifetime prevalence and age-of-onset distributions of DSM-IV disorders in the National Comorbidity Survey Replication. Arc Gen Psychiatry 62: 593-602.

Kessler RC, Wittchen HU (2002). Patterns and correlates of generalized anxiety disorder in community samples. J Clin Psychiatry 63(Suppl 8): 4-10.

Klemenhagen KC, Gordon JA, David DJ, Hen R, Gross CT (2006). Increased fear response to contextual cues in mice lacking the 5-HT1A receptor. Neuropsychopharmacology 31: 101-111.

Knudsen EI (2004). Sensitive periods in the development of the brain and behavior. J Cogn Neurosci 16: 1412-1425.

Loizou LA (1972). The postnatal ontogeny of monoaminecontaining neurones in the central nervous system of the albino rat. Brain Res 40: 395-418.

Moffitt TE, Caspi A, Harrington H, Milne BJ, Melchior M, Goldberg $\mathrm{D}$ et al (2007). Generalized anxiety disorder and depression: childhood risk factors in a birth cohort followed to age 32 . Psychol Med 37: 441-452. Prospective study demonstrating that early life risk factors are overrepresented in people with a lifetime anxiety disorder and co-morbid anxiety and depression but not in those with depression alone.

Murrin LC, Sanders JD, Bylund DB (2007). Comparison of the maturation of the adrenergic and serotonergic neurotransmitter systems in the brain: implications for differential drug effects on juveniles and adults. Biochem Pharmacol 73: 1225-1236.

Nakamura K, Koyama Y, Takahashi K, Tsurui H, Xiu Y, Ohtsuji M et al (2006). Requirement of tryptophan hydroxylase during development for maturation of sensorimotor gating. J Mol Biol 363: 345-354.

Ninan PT, Berger J (2001). Symptomatic and syndromal anxiety and depression. Depress Anxiety 14: 79-85.

Nolen-Hoeksema S, Girgus JS, Seligman MEP (1992). Predictors and consequences of childhood depressive symptoms a 5-year longitudinal study. J Abnorm Psychol 101: 405-422.

Parker G, Wilhelm K, Mitchell P, Austin MP, Roussos J, Gladstone $\mathrm{G}$ (1999). The influence of anxiety as a risk to early onset major depression. J Affect Disord 52: 11-17.

Parks CL, Robinson PS, Sibille E, Shenk T, Toth M (1998). Increased anxiety of mice lacking the serotonin1A receptor. Proc Natl Acad Sci USA 95: 10734-10739.

Pokorny J, Yamamoto T (1981). Postnatal ontogenesis of hippocampal CA1 area in rats. II. Development of ultrastructure in stratum lacunosum and molecular. Brain Res Bull 7: 121-130.

Ramboz S, Oosting R, Amara DA, Kung HF, Blier P, Mendelsohn M et al (1998). Serotonin receptor 1A knockout: an animal model of anxiety-related disorder. Proc Natl Acad Sci USA 95: 14476-14481.

Roy MA, Neale MC, Pedersen NL, Mathe AA, Kendler KS (1995). A twin study of generalized anxiety disorder and major depression. Psychol Med 25: 1037-1049.

Santarelli L, Saxe M, Gross C, Surget A, Battaglia F, Dulawa S et al (2003). Requirement of hippocampal neurogenesis for the behavioral effects of antidepressants. Science 301: 805-809.

Schwartz CE, Snidman N, Kagan J (1999). Adolescent social anxiety as an outcome of inhibited temperament in childhood. J Am Acad Child Adolesc Psychiatry 38: 1008-1015.

Sullivan PF, Neale MC, Kendler KS (2000). Genetic epidemiology of major depression: review and meta-analysis. Am J Psychiatry 157: $1552-1562$.

Tansey EP, Chow A, Rudy B, McBain CJ (2002). Developmental expression of potassium-channel subunit Kv3.2 within subpopulations of mouse hippocampal inhibitory interneurons. Hippocampus 12: 137-148.

Van Ameringen M, Mancini C, Oakman JM (1998). The relationship of behavioral inhibition and shyness to anxiety disorder. J Nerv Ment Dis 186: 425-431.

Weissman MM, Wickramaratne P, Nomura Y, Warner V, Verdeli $\mathrm{H}$, Pilowsky DJ et al (2005). Families at high and low risk for depression: a 3-generation study. Arch Gen Psychiatry 62: 29-36. Demonstrates that anxiety disorders are the early markers of psychopathology in families with familial depression.

Westerga J, Gramsbergen A (1990). The development of locomotion in the rat. Brain Res Dev Brain Res 57: 163-174.

Wittchen HU, Kessler RC, Pfister H, Lieb M (2000). Why do people with anxiety disorders become depressed? A prospectivelongitudinal community study. Acta Psychiatr Scand 102(Suppl): $14-23$. 